\title{
Vaginal-Rectal Colonization With Group A Streptococci in Late Pregnancy
}

\author{
Philip B. Mead ${ }^{1 *}$ and Washington C. Winn ${ }^{2}$ \\ ${ }^{1}$ Department of Obstetrics and Gynecology, University of Vermont College of Medicine, \\ Burlington, VT \\ ${ }^{2}$ Department of Pathology, University of Vermont College of Medicine, Burlington, VT
}

\begin{abstract}
Objective: To determine the vaginal-rectal colonization rate with group A streptococci in late pregnancy.

Methods: All patients delivering at a northern New England hospital over a 38 month period had 35-37 week vaginal-rectal swabs cultured for group A and group B streptococci, using selective media and slide agglutination.

Results: Six thousand nine hundred forty-four screening cultures were obtained. Among these 1,393 were positive for group B streptococci and 2 for group A streptococci, yielding colonization rates of $20.1 \%$ and $0.03 \%$, respectively.

Conclusions: Vaginal-rectal colonization with group A streptococci is rare, arguing against the need for establishing group A streptococcal screening programs in pregnancy. An approach for managing this uncommon finding is presented. Infect. Dis. Obstet. Gynecol. 8:217-219, 2000. (c) 2000 Wiley-Liss, Inc.
\end{abstract}

KEY WORDS

group A beta-hemolytic streptococcus; vaginal flora; slide agglutination

A lthough group B streptococci (GBS) have been described as common members of the vaginal flora for over 60 years, vaginal colonization with group A streptococci (GAS) has been reported infrequently. Because of recent concern over the increasing incidence of severe, invasive GAS infections, questions have been raised regarding the value of instituting antepartum screening programs for GAS. Knowledge of GAS colonization rates in late pregnancy would help to inform these discussions.

In 1996 the Centers for Disease Control and Prevention (CDC) issued guidelines for prevention of group B streptococcal neonatal sepsis, including recommendations for vaginal-rectal screening cultures for GBS at 35-37 weeks of gestation. ${ }^{1}$ At the University of Vermont/Fletcher Allen Health Care, this protocol was reliably in place by late 1996, and the laboratory methods used included testing of all presumptive beta-hemolytic colonies for both group A and group B antigen, allowing us to report our 3 year experience with vaginal GAS colonization in late pregnancy.

\section{SUBJECTS AND METHODS}

UVM/FAHC Medical Center, the only hospital in Vermont's largest city, performs approximately 2,300 deliveries annually. Patients are drawn from all of the private practices, faculty practices, and clinics in Chittenden County. Beginning in the fall of 1996, all patients underwent a 35-37 week vaginal-rectal screening culture for GBS as recommended in the CDC culture-based protocol. Rayon-tipped swabs of the vaginal introitus and

*Correspondence to: Philip B. Mead, MD, Department of Obstetrics and Gynecology, University of Vermont College of Medicine, Shepardson 618, 111 Colchester Avenue, Burlington, VT 05401. E-mail: phil.mead@vtmednet.org 
anorectum were sent to the on-site laboratory in a nonnutritive moist swab transport system (Culturette II; Becton Dickinson, Sparks, MD). The vaginal/rectal swabs were inoculated onto sheep blood agar, colistin-naladixic acid agar, and into selective Todd-Hewitt broth (Lim broth). After incubation, the broth was subcultured to an additional sheep blood agar plate, unless group B beta hemolytic streptococci had been identified on the primary plates. This subcultured plate was incubated for an additional $24 \mathrm{hr}$. All beta-hemolytic streptococci were tested for group A and group B antigens using slide agglutination (Streptex; Abbott Laboratories, Abbott Park, IL).

We reviewed all 35-37 week screening cultures obtained from January 1, 1997, through February 29, 2000, for isolations of GAS and GBS. Medical records of patients with positive GAS isolations were reviewed for management and outcome.

\section{RESULTS}

Six thousand nine hundred forty-four 35-37 week vaginal-rectal screening cultures were obtained during the 38 month study period. Among these, 1,393 were positive for GBS and 2 were positive for GAS, yielding colonization rates of $20.1 \%$ for GBS and $0.03 \%$ for GAS.

The two positive GAS cultures were obtained in March, 1997, and February, 2000. Both patients were GBS-negative. Neither received antepartum treatment, and both received standard GBS intrapartum prophylaxis with penicillin. Neither of the mothers or infants became infected.

\section{DISCUSSION}

For this population, during the time period studied, 35-37 week vaginal-rectal colonization with GAS was extremely uncommon, occurring only once in every 3,472 deliveries. Larsen ${ }^{2}$ has reviewed the literature pertaining to the microbiology of the female genital tract and concluded that "only rarely" are GAS stable members of the normal vaginal flora. A classic study of cervical flora during pregnancy and the puerperium found no group A beta-hemolytic streptococci, supporting the concept that these organisms rarely colonize the lower genital tract. ${ }^{3}$ To our knowledge, ours is the first study to determine systematically the vaginal-rectal GAS colonization rate in a large number of women in late pregnancy. Validation of the rarity of GAS colonization is important, we believe; it argues against the need for development of GAS screening programs in pregnancy.

This is a northern New England population of diverse socioeconomic status who are almost exclusively white. Whether these results are generalizable to other populations is unknown. Orogenital sex is a postulated mechanism for female genital tract colonization with GAS. We have no data on the frequency of this practice in our population but have no reason to suspect it differs widely from that among other predominantly white U.S. populations. Oral sex is known to be less common among nonwhite populations, and multivariate analyses confirm the independent effect of race on this practice. ${ }^{4}$ If this indeed is an important mechanism for female genital colonization, our mostly white population might be expected to have a higher incidence of colonization with GAS. The colonization rate for GBS in this study, $20.1 \%$, is well within the rates of $18-26 \%$ reported by other investigators. ${ }^{5-7}$

Perinatally acquired infection from GAS has been described infrequently, ${ }^{8}$ a fact that is not surprising given the low colonization rate described here. Most infectious disease authorities view GAS as a greater threat than GBS for the initiation of infection, and, as noted above, question whether it ever can be considered as "normal flora." The increasing frequency of potentially catastrophic invasive GAS infections, including streptococcal toxic shock syndrome and necrotizing fasciitis, ${ }^{9-13}$ argues for thoughtfulness in responding to the identification of GAS from any culture, including that from the lower genital tract. Until more data are available, we have recently instituted a policy of treating all positive GAS 35-37 week rectovaginal screening cultures (penicillin-VK $500 \mathrm{mg}$ p.o. QID $\times 10$ days) and performing a test-of-cure culture. In addition, standard intrapartum prophylaxis with penicillin $G$ is used. Although this is not evidencebased, it may provide prophylaxis against a serious invasive GAS infection, and the infrequency of use would seem to obviate concerns for antibiotic selection pressure. In summary, we have documented that vaginal-rectal colonization with GAS in late pregnancy is a rare event, and we conclude that establishing GAS screening programs in pregnancy is not warranted. 


\section{REFERENCES}

1. Centers for Disease Control. Prevention of perinatal group B streptococcal disease: a public health perspective. MMWR 1996;45(RR-7):1-24.

2. Larsen B. Microbiology of the female genital tract. In: Pastorek JG, editor. Obstetric and gynecologic infectious disease. New York: Raven Press; 1994. p 11-25.

3. Goplerud CP, Ohm MJ, Galask RP. Aerobic and anaerobic flora of the cervix during pregnancy and the puerperium. Am J Obstet Gynecol 1976;126:858-868.

4. Laumann EO, Gagnon JH, Michael RT, Michaels S. The social organization of sexuality. Chicago: The University of Chicago Press; 1994. p 98.

5. Yancey MK, Duff P, Clark P, Kurtzer T, Frentzen BH, Kubilis $P$. Peripartum infection associated with vaginal group B streptococcal colonization. Obstet Gynecol 1994;84:816-819.

6. Yancey MK, Schuchat A, Brown LK, Ventura VL, Markenson GR. The accuracy of late antenatal screening cultures in predicting genital group B streptococcal colonization at delivery. Obstet Gynecol 1996;88:811815.
7. Regan JA, Klebanoff MA, Nugent RP. The epidemiology of group B streptococcal colonization in pregnancy. Obstet Gynecol 1991;77:604-610.

8. Panaro NR, Lutwick LI, Chapnick EK. Intrapartum transmission of group A streptococcus. Clin Infect Dis 1993;17:79-81.

9. Dotters DJ, Katz VL. Streptococcal toxic shock associated with septic abortion. Obstet Gynecol 1991;78:549551.

10. Silver RM, Heddleston LN, McGregor JA, Gibbs RS. Life-threatening infection due to group A streptococci. Obstet Gynecol 1992;79:894-896.

11. Nathan L, Peters MT, Ahmed AM, Leveno KJ. The return of life-threatening puerperal sepsis caused by group A streptococci. Am J Obstet Gynecol 1993;169: 571-572.

12. Rowan JA, North RA. Necrotizing fasciitis in the puerperium. Am J Obstet Gynecol 1995;173:241-242.

13. Udagawa $H$, Oshio $Y$, Shimizu Y. Serious group A streptococcal infection around delivery. Obstet Gynecol 1999;94:153-157. 


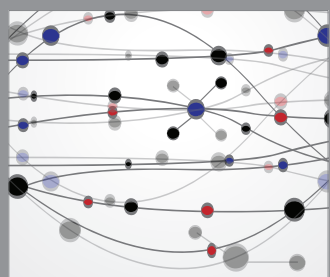

The Scientific World Journal
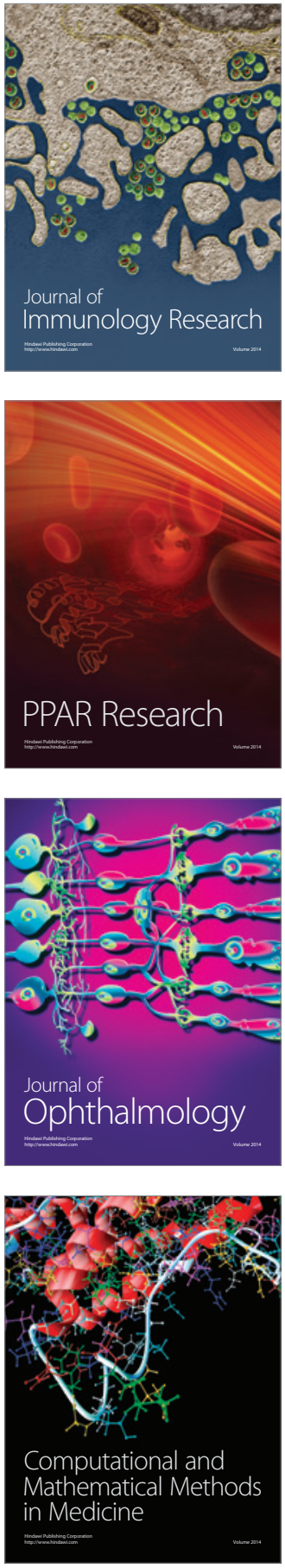

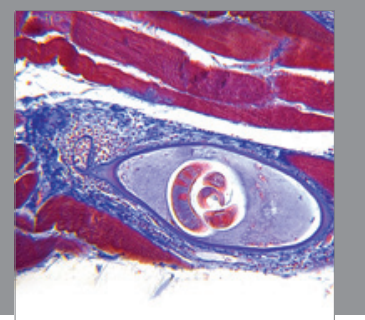

Gastroenterology

Research and Practice
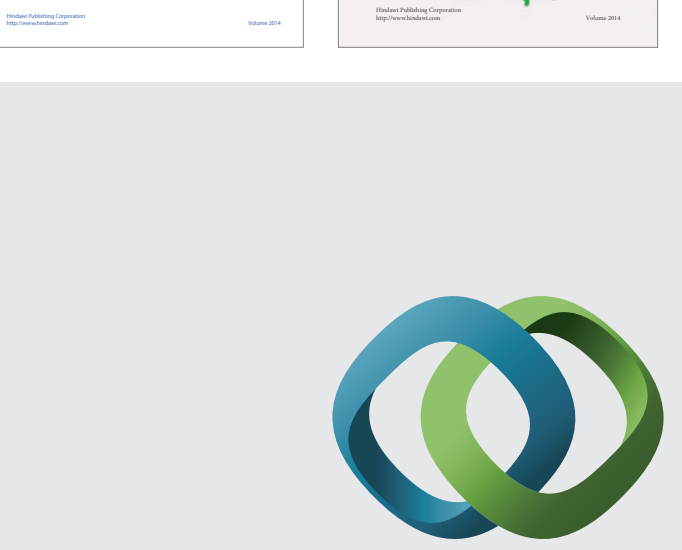

\section{Hindawi}

Submit your manuscripts at

http://www.hindawi.com
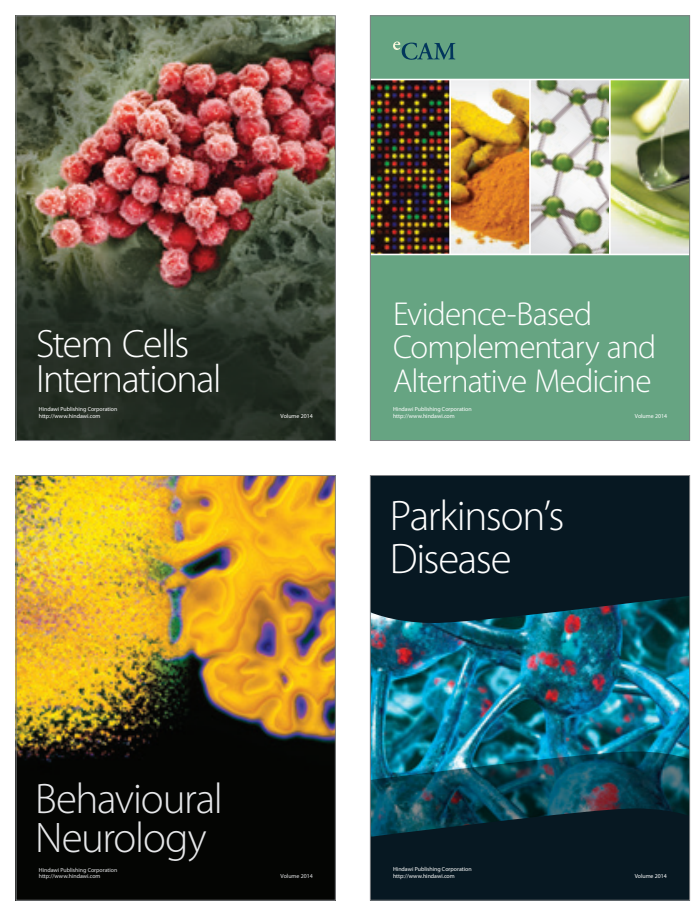

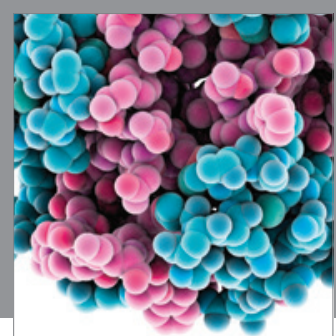

Journal of
Diabetes Research

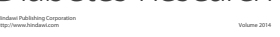

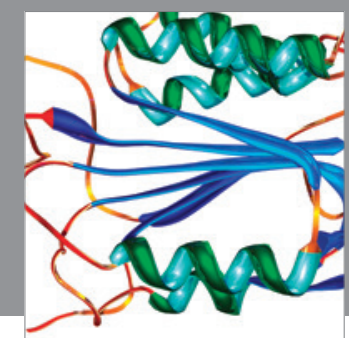

Disease Markers
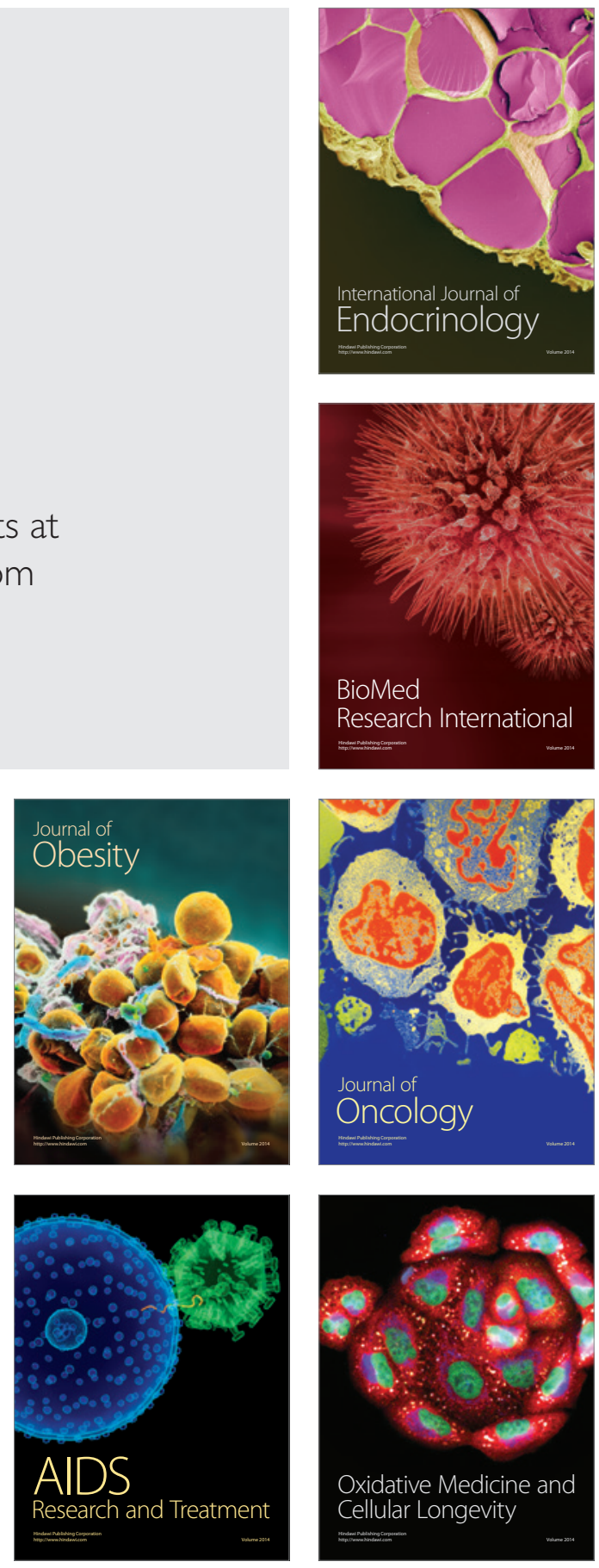